\title{
Identification of a missense mutation in the tyrosinase gene in a Chinese family with oculocutaneous albinism type 1
}

\author{
QIAN LU ${ }^{1 *}$, LAMEI YUAN $^{1 *}$, HONGBO XU $^{1}$, XIANGJUN HUANG ${ }^{1,2}$, \\ ZHIJIAN YANG ${ }^{1}$, JUNHUI YI ${ }^{3}, \mathrm{BIN} \mathrm{NI}^{4}, \mathrm{YONG} \mathrm{CHEN}^{4}$ and $\mathrm{HAO} \mathrm{DENG}^{1,5}$ \\ ${ }^{1}$ Center for Experimental Medicine, The Third Xiangya Hospital, Central South University, Changsha, Hunan 410013; \\ ${ }^{2}$ Department of General Surgery, The First Hospital of Hunan University of Chinese Medicine, Changsha, Hunan 410007; \\ ${ }^{3}$ Department of Ophthalmology, The Third Xiangya Hospital, Central South University, Changsha, Hunan 410013; \\ ${ }^{4}$ Key Laboratory of Genetics and Birth Health of Hunan Province, Family Planning Institute of Hunan Province, \\ Changsha, Hunan 410078; ${ }^{5}$ Department of Neurology, The Third Xiangya Hospital, \\ Central South University, Changsha, Hunan 410013, P.R. China
}

Received December 8, 2015; Accepted December 7, 2016

DOI: $10.3892 / \mathrm{mmr} .2017 .6137$

\begin{abstract}
Oculocutaneous albinism (OCA) is a group of heterogeneous and autosomal recessive disorders characterized by a reduction or complete loss of melanin biosynthesis in melanocytes. OCA type 1 (OCA1) is the most severe and common form of OCA, and is caused by mutations in the tyrosinase gene $(T Y R)$. The present study aimed to identify the genetic cause of OCA1 in a four-generation consanguineous Chinese Han family. Complete physical examinations were performed and blood samples were collected from five members of the family and 100 unrelated healthy controls. Exome sequencing was conducted in the proband, followed by verification in other family members, using Sanger sequencing. Patients in the family presented with typical OCA1 features, including hypopigmentation of the skin and hair, and distinctive ocular changes. A homozygous missense variant, c.896G >A (p.R299H), in the TYR gene was identified in two patients, which co-segregated with disease in the family. This variant was not present in the 100 healthy controls. These results expand the number of mutations identified to be responsible for OCA1 in the Chinese Han
\end{abstract}

Correspondence to: Professor Hao Deng, Center for Experimental Medicine, The Third Xiangya Hospital, Central South University, 138 Tongzipo Road, Changsha, Hunan 410013, P.R. China E-mail: hdeng008@yahoo.com

Professor Yong Chen, Key Laboratory of Genetics and Birth Health of Hunan, Family Planning Institute of Hunan, 1292 Yuanda Road, Changsha, Hunan 410078, P.R. China

E-mail: chenyong0008@sina.com

*Contributed equally

Key words: missense mutation, oculocutaneous albinism, the tyrosinase gene, tyrosinase population, and may have implications for genetic counseling and clinical management of the disease.

\section{Introduction}

Oculocutaneous albinism (OCA) is a group of heterogeneous and autosomal recessive disorders characterized by a reduction or complete loss of melanin biosynthesis in melanocytes (1). All types of OCA demonstrate a lack of melanin pigment in the skin, hair and eyes, and are often accompanied by ocular abnormalities, including varying degrees of nystagmus, hypopigmentation of the iris, foveal hypoplasia, poor vision and refractive errors, photophobia and occasionally color vision impairment (2). The prevalence of OCA subtypes varies considerably between ethnicities. OCA has a prevalence of $\sim 1: 20,000$ individuals worldwide, or $\sim 1: 18,000$ in the Chinese Han population (3). At least 17 genes, including four non-syndromic and 13 syndromic, have been identified to be associated with OCA. The tyrosinase (TYR), oculocutaneous albinism II, tyrosinase-related protein 1 and solute carrier family 45, member 2 genes are the four non-syndromic OCA genes and are responsible for OCA type 1 (OCA1; MIM 203100), type 2 (OCA2; MIM 203200), type 3 (OCA3; MIM 203290), and type 4 (OCA4; MIM 606574), respectively. The 13 syndromic OCA genes include the Hermansky-Pudlak syndrome 1 (HPS1), adaptor-related protein complex 3, $\beta 1$ subunit, HPS3, HPS4, HPS5, HPS6, dystrobrevin binding protein 1 , biogenesis of lysosomal organelles complex-1 subunit 3 (BLOC1S3), BLOC1S6, lysosomal trafficking regulator, myosin VA, ras-related protein and melanophilin genes $(4,5)$. Due to the phenotypic variation and overlap in clinical presentation among different OCA subtypes, genetic analysis is essential to identify the gene defect and the subtype of OCA (6).

OCA1 is the most severe and common form of OCA, and is caused by mutations of the TYR gene (7). It is often divided into two categories: OCA1A and OCA1B. Patients with OCA1A are characterized by a lifelong and complete absence of pigment in the skin, hair and eyes. Patients with OCA1B 
may have a reduced activity of tyrosinase and may produce some melanin over time, resulting in a darkening of hair $(3,8)$. OCA1 affects $\sim 1$ in 40,000 individuals in the majority of populations, and has been reported to be the most common subtype of OCA in Japanese, non-Hispanic Caucasian and Danish populations (9). It accounts for $\sim 64.3 \%$ of patients with OCA in China. However, OCA1 is rare among African-Americans, reflecting a population-specific distribution of different OCA subtypes (6).

The present study aimed to identify the genetic mutation responsible for OCA1 in a four-generation family of Chinese origin. Patients in the family presented with typical OCA1 characteristics, including white hair and skin, nystagmus, impaired visual acuity, photophobia, color vision impairment, refractive errors, foveal hypoplasia and iris hypopigmentation and translucency. The p.R299H variant of the TYR gene, predicted to disrupt the overall integrity of tyrosinase, was revealed to co-segregate with patients in this family and was absent in healthy controls, indicating that it is a pathogenic mutation.

\section{Materials and methods}

Subjects. A four-generation, 11-member consanguineous Chinese Han family with OCA from Hunan, China was recruited from The Third Xiangya Hospital, Central South University (Hunan, China; Fig. 1). Blood samples from the five living members of the family, including two affected patients (IV:2 and IV:3) and three unaffected members (III:1, III:2 and IV:1) were collected. Additionally, blood samples were collected from 100 unrelated healthy controls (male, $\mathrm{n}=50$; female, $\mathrm{n}=50$; age, $45.8 \pm 3.4$ years) with normal pigmentation and with no family history of ocular abnormalities, from the same region of China. All participants provided written informed consent. The present study was approved by the Institutional Review Board of The Third Xiangya Hospital, Central South University.

Exome capture. Genomic DNA was extracted from peripheral blood samples according to the standard phenol-chloroform extraction method (10). Exome capture was performed on genomic DNA from the proband (Fig. 1) by the Novogene Bioinformatics Institute (Beijing, China). The SureSelect Human All Exon V5 kit (Agilent Technologies, Inc., Santa Clara, CA, USA) was used for exome capture and the HiSeq 2000 platform (Illumina, Inc., San Diego, CA, USA) was used for sequencing, following the manufacturer's protocol. A total of $1.5 \mu \mathrm{g}$ genomic DNA was used to construct the exome library and the genomic DNA was sheared into 180-280 bp for enrichment. Enrichment libraries for target regions were sequenced by the HiSeq 2000 platform, which generated 100 bp pair-end reads.

Read mapping and variant analysis. All the clean reads were aligned to the human reference genome (University of California, Santa Cruz; Build 37.1, hg19) using Burrows-Wheeler Aligner (bio-bwa.sourceforge.net). High quality alignment was required to guarantee variant calling accuracy $(>0)$ to detect single nucleotide polymorphisms (SNPs) and insertions-deletions (indels). Picard software

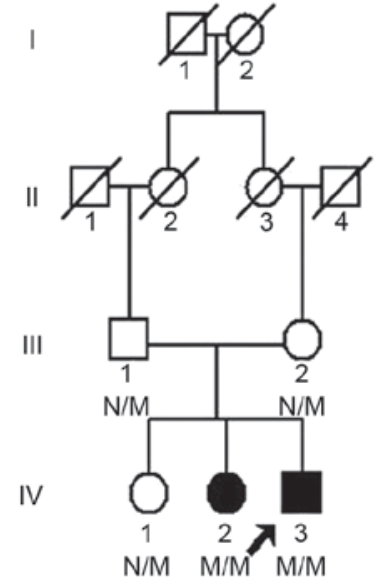

Figure 1. Pedigree of the family with oculocutaneous albinism type 1. Filled symbols represent affected members; empty symbols represent unaffected members. Squares, males; circles, females. Symbols with a diagonal line represent deceased family members. The proband is indicated by an arrow. N, normal; M, TYR c.896G >A (p.R299H) mutation.

(sourceforge.net/projects/picard/), the Genome Analysis Toolkit software version 2.1 (software.broadinstitute. org/gatk/) and SAMtools (samtools.sourceforge.net) were used for base quality recalibration. Following this, the binary alignment/map results were obtained, ready for analysis, and ANNOVAR software (Annotate Variation; annovar. openbioinformatics.org/enllatest/user-guider/download) was used to annotate SNPs and indels. Variant lists were filtered against the dbSNP build 137 (dbSNP137), database of SNPs (www.ncbi.nlm.nih.gov/projects/SNP/snp_summary.cgi), the 1000 Genomes Project (1000genomes release_20100804; www.1000genomes.org/) and the National Heart, Lung and Blood Institute Exome Sequencing Project (NHLBI ESP) 6500. Polymorphism Phenotyping version 2 (PolyPhen-2; genetics.bwh.harvard.edu/pph2/) and Sorting Intolerant from Tolerant (SIFT; sift.jcvi.org/) software were used to predict protein functions.

Mutation validation. Sanger sequencing was used to confirm the presence and identity of potential disease-causing variants, using the 3500 Genetic Analyzer sequencer (Applied Biosystems; Thermo Fisher Scientific, Inc., Waltham, MA, USA). Polymerase chain reaction amplification and Sanger sequencing were conducted as described previously (11), using the following primers: Forward, 5'-GTCTGTAGC CGATTGGAGGA-3' and reverse, 5'-GCAGCTTTATCC ATGGAACC-3'.

The National Centre for Biotechnology Information Basic Local Alignment Search Tool (blast.ncbi.nlm.nih.gov/Blast. cgi) was used to perform multiple sequence alignments. The pathogenic potential of amino acid changes as possible disease-causing mutations was further examined using the MutationTaster online tool (http://www.mutationtaster.org/).

\section{Results}

Clinical findings. Complete physical examinations, including detailed ophthalmic examination (best-corrected visual acuity 
Table I. Clinical and genetic data of the two patients with the tyrosinase gene c.896G >A (p.R299H) mutation.

\begin{tabular}{lcc}
\hline Parameter & IV:2 & IV:3 \\
\hline Gender & Female & Male \\
Age (years) & 42 & 39 \\
Genotype & Homozygous & Homozygous \\
Hair color (at birth/at present) & White/white & White/white \\
Skin color & White & White \\
Nystagmus & + & + \\
Iris hypopigmentation and & + & + \\
translucency & & \\
Foveal hypoplasia & + & + \\
Refractive errors & + & + \\
Color vision impairment & + & + \\
Photophobia & + & + \\
Impaired visual acuity & + & + \\
\hline
\end{tabular}

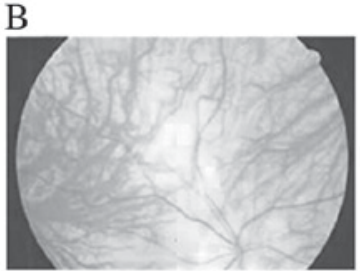

OD

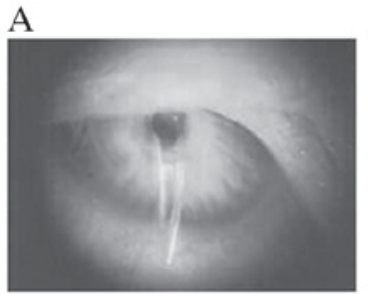

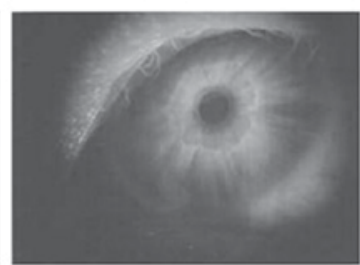

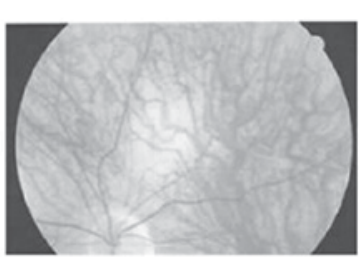

OS
Figure 2. Representative images of the clinical characteristics of the proband (IV:3). Images demonstrate (A) iris hypopigmentation and translucency in the anterior segment and (B) sunset-glow fundus with visible choroidal vessels in the fundus. OD, Oculus Dexter; OS, Oculus Sinister.

testing, slit-lamp examination, dilated fundus examination and optical coherence tomography) were performed. The two patients had typical OCA skin, hair and eye symptoms; they presented with white hair that had not altered in color over time. More detailed descriptions of clinical features of the two OCA patients are presented in Table I. The proband (IV:3) presented with evident horizontal nystagmus, photophobia, impaired visual acuity, absence of the foveal pit, visible choroidal macula vessels, and iris hypopigmentation and translucency (Fig. 2). Additionally, he had refractive errors (-3.00D) and color vision impairment.

Mutation screening. Exome sequencing of the proband (IV:3; Fig. 1) was performed in the Chinese family with OCA. A total of 46.37 million reads with an average read length of $100 \mathrm{bp}$ were generated from the patient; 46.33 million bases (99.92\%) were aligned to the human reference sequence. A total of

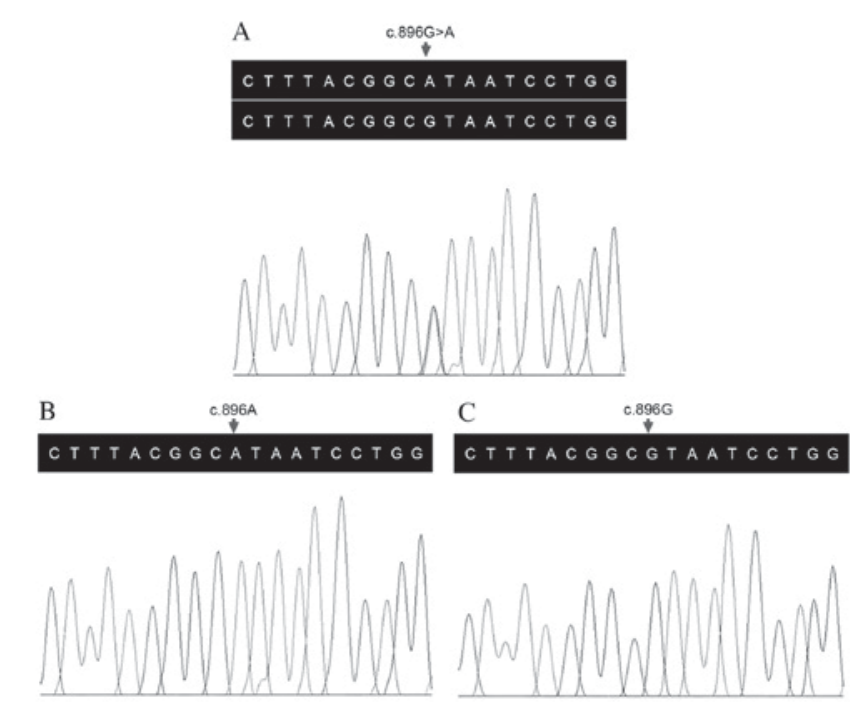

Figure 3. Tyrosinase gene DNA sequencing analysis of p.R299H mutation. (A) A heterozygous p.R299H mutation in the parent of the patient (III:1) and (B) a homozygous p.R299H mutation in the proband (IV:3). (C) Unrelated healthy control.

\begin{tabular}{ll} 
& \multicolumn{2}{c}{ p.R299H } \\
Human & NGTPEGPLRRNPGNHDKSR \\
Chimpanzee & NGTPEGPLRRNPGNHDKSR \\
Rhesus monkey & NGMPEGPLRRNPGNHDKR \\
Cattle & NGTSEGPLLRNPGNHDKAR \\
Sheep & NGTSEGPLLRNPGNHDKAR \\
Dog & DGTPEGPLLRNPGNHDKAR \\
House mouse & DGTPEGPLLRNPGNDKAK \\
Norway rat & DGTPEGPLLRNPGNHDKAK \\
Chicken & NATSEGPILRNPGNNDKSR \\
Zebrafish & DGSPEGPLLNPGDHDRIR
\end{tabular}

Figure 4. Conservation analysis of the tyrosinase p.R299 amino acid residue. The arrow indicates that arginine at position 299 was phylogenetically conserved among various species.

38,626 genetic variants in the coding regions and splice sites, and 36,091 SNPs, including 17,241 in the exon regions and 1,595 in the splice sites, were identified. Furthermore, 2,535 indels, including 410 in the exon regions and 190 in the splice sites, were detected. A prioritization scheme was used to detect the potential pathogenic mutation in the patient, as previously described (12). Known variants identified in the dbSNP137, 1,000 Genomes Project and NHLBI ESP 6500 with a minor allele frequency $>0.50 \%$ were excluded. SIFT and PolyPhen-2 were used to predict the function of non-synonymous variants. By applying the above filtration criteria, the number of candidate genes was reduced by $>99.03 \%$; only 238 novel variants were identified to be potentially disease-causing, therefore were selected for further analysis.

The parents of the proband were identified to carry a heterozygous variant in the TYR gene (Fig. 3A). A c.896G $>\mathrm{A}$ (p.R299H) variant in the two alleles of the $T Y R$ gene was identified in the proband (Fig. 3B) following validation by Sanger sequencing. One female patient (IV:2) in the family was subsequently identified to carry the same homozygous 
mutation. This variant co-segregated with disease phenotype. The variant was absent in the control cohort, consisting of 100 ethnicity-matched unrelated controls (Fig. 3C). Arginine at position 299 was phylogenetically conserved among various species (Fig. 4). MutationTaster predicted that the substitution was disease-causing. These data indicated that the $T Y R$ p.R299H variant may be the disease-associated variant in the family investigated in the present study.

\section{Discussion}

Albinism was one of the earliest genetic disorders to be studied. In 1903, Farabee (13) first suggested that human albinism may be recessively inherited. The mouse and human TYR genes were isolated in 1987. In 1989, the first mutation (nonsense mutation) in the $T Y R$ gene responsible for human OCA was reported by Tomita et al (14). There are 349 TYR sequence variants recorded in the Human Gene Mutation Database (www.hgmd.cf.ac.uk/ac/index.php). A wide spectrum of mutations, including gross and small deletions, small insertions, small indels, and splicing, missense and nonsense mutations, have been described, with missense mutations being the most common.

The TYR gene (MIM 606933) is located on chromosome 11q14.3, and contains 5 exons spanning $\sim 65 \mathrm{~kb}$ of genomic DNA. It encodes a 529-amino acid protein, tyrosinase. Tyrosinase consists of an 18-amino acid signal peptide, two copper binding sites, a transmembrane region at the C-terminal end and an epidermal growth factor-like motif (15). It is a glycoprotein and a copper-containing oxidase, and is expressed in melanocytes. Tyrosinase serves an important role in melanin biosynthesis by catalyzing the rate-limiting conversions of tyrosine to dihydroxy-phenylalanine (DOPA) and from DOPA to DOPA-quinone. In addition, it may catalyze the conversion of 5,6-dihydroxyindole to indole-5,6 quinone (16). Mutations in the TYR gene lead to decreased or even absent tyrosinase enzyme activity, and subsequently, a decreased or complete loss of melanin synthesis. Numerous missense mutations are located in or adjacent to the copper binding sites, and interrupt the normal function of tyrosinase, by affecting copper binding or by disrupting the substrate binding site (17).

The present study examined a Chinese Han family with OCA1, characterized by white hair that had not altered in color with age, white skin, nystagmus, impaired visual acuity, photophobia, color vision impairment, refractive errors, foveal hypoplasia and iris hypopigmentation and translucency. The homozygous c.896G >A variant (p.R299H, rs61754375) in the TYR gene was identified in the two patients in the family and was absent in the 100 unrelated ethnicity-matched controls. The mutation was predicted to be 'probably damaging' by PolyPhen-2, 'damaging' by SIFT and 'disease-causing' by MutationTaster. These results suggested that the c.896G >A variant (p.R299H) may be pathogenic. Multiple sequence alignment demonstrated that the arginine residue is highly conserved among vertebrates, suggesting that it may be important for function. The parents of the patients, with a heterozygous p.R299H mutation in the $T Y R$ gene, presented a typical phenotype with no symptoms of OCA. Notably, homozygous and heterozygous p.R299H mutations in the $T Y R$ gene have been frequently identified in Chinese, Caucasian, Korean and Arabian patients with OCA1, indicating that this location is a mutation hotspot in OCA1 (7,17-20).

Albinism is understood to occur in the majority of fish, birds, reptiles, amphibians and mammals (21). Molecular abnormalities in the Tyr gene that are associated with OCA have been described in whales, chickens, mice, rats, cats, rabbits, cattle, buffalo, American mink, ferrets and rhesus monkeys (22). In 2005, Blaszczyk et al (23) identified the mutation p.R299H in the albino Wistar rat, emphasizing the functional significance of this mutation leading to OCA.

In conclusion, the results of the present study confirmed the clinical diagnosis of OCA1 in a Chinese family by identifying a p.R299H mutation in the TYR gene. These findings suggested that exome sequencing may be a cost-effective tool for accurate diagnosis of the disease, therefore providing genetic counseling options for individuals with OCA. Further studies using appropriate $T Y R$-deficient animal models may facilitate the development of novel therapeutic strategies for the treatment of this disease.

\section{Acknowledgements}

The authors thank the participating subjects for their cooperation; and investigators for collecting clinical, radiological and genetic information and DNA specimens. The present study was supported by the Talent Projects of New Xiangya (to H.D.).

\section{References}

1. Kamaraj B and Purohit R: Mutational analysis of oculocutaneous albinism: A compact review. Biomed Res Int 2014: 905472, 2014.

2. Khordadpoor-Deilamani F, Akbari MT, Karimipoor M and Javadi G: Sequence analysis of tyrosinase gene in ocular and oculocutaneous albinism patients: Introducing three novel mutations. Mol Vis 21: 730-735, 2015.

3. Wang Y, Wang Z, Chen M, Fan N, Yang J, Liu L, Wang Y and Liu X: Mutational analysis of the TYR and OCA2 genes in four Chinese families with oculocutaneous albinism. PLoS One 10: e0125651, 2015.

4. Ghodsinejad Kalahroudi V, Kamalidehghan B, Arasteh Kani A, Aryani O, Tondar M, Ahmadipour F, Chung LY and Houshmand M: Two novel tyrosinase (TYR) gene mutations with pathogenic impact on oculocutaneous albinism type 1 (OCA1). PLoS One 9: e106656, 2014.

5. Gargiulo A, Testa F, Rossi S, Di Iorio V, Fecarotta S, de Berardinis T, Iovine A, Magli A, Signorini S, Fazzi E, et al: Molecular and clinical characterization of albinism in a large cohort of Italian patients. Invest Ophthalmol Vis Sci 52: 1281-1289, 2011.

6. Grønskov K, Ek J and Brondum-Nielsen K: Oculocutaneous albinism. Orphanet J Rare Dis 2: 43, 2007.

7. Wang Y, Guo X, Li W and Lian S: Four novel mutations of TYR gene in Chinese OCA1 patients. J Dermatol Sci 53: 80-81, 2009.

8. Wei AH, Yang XM, Lian S and Li W: Genetic analyses of Chinese patients with digenic oculocutaneous albinism. Chin Med J (Engl) 126: 226-230, 2013.

9. Liu N, Kong XD, Shi HR, Wu QH and Jiang M: Tyrosinase gene mutations in the Chinese Han population with OCA1. Genet Res (Camb) 96: e14, 2014.

10. Guo Y, Song Z, Xu H, Yi J, Zheng W, Xiang H, Deng X, Lv H, Gao K, Qi Y and Deng H: Heterogeneous phenotype in a family with the FERM domain-containing 7 gene R335X mutation. Can J Ophthalmol 49: 50-53, 2014.

11. Yuan L, Wu S, Xu H, Xiao J, Yang Z, Xia H, Liu A, Hu P, Lu A, Chen Y, et al: Identification of a novel PHEX mutation in a Chinese family with X-linked hypophosphatemic rickets using exome sequencing. Biol Chem 396: 27-33, 2015. 
12. Zheng W, Chen H, Deng X, Yuan L, Yang Y, Song Z, Yang Z, Wu Y and Deng H: Identification of a novel mutation in the Titin gene in a Chinese family with limb-girdle muscular dystrophy $2 \mathrm{~J}$. Mol Neurobiol 53: 5097-5102, 2016.

13. Farabee WC: Notes on Negro albinism. Science 17: 75, 1903.

14. Tomita Y, Takeda A, Okinaga S, Tagami H and Shibahara S: Human oculocutaneous albinism caused by a single base insertion in the tyrosinase gene. Biochem Biophys Res Commun 164: 990-996, 1989

15. Oetting WS and King RA: Molecular basis of albinism: Mutations and polymorphisms of pigmentation genes associated with albinism. Hum Mutat 13: 99-115, 1999.

16. Shah SA, Din SU, Raheem N, Daud S, Mubeen J, Nadeem A, Tayyab M, Baloch DM, Babar ME and Ahmad J: Identification of a novel mutation (p.Ile198Thr) in gene TYR in a Pakistani family with nonsyndromic oculocutaneous albinism. Clin Exp Dermatol 39: 646-648, 2014.

17. Liu J, Choy KW, Chan LW, Leung TY, Tam PO, Chiang SW, Lam DS, Pang CP and Lai TY: Tyrosinase gene (TYR) mutations in Chinese patients with oculocutaneous albinism type 1 . Clin Exp Ophthalmol 38: 37-42, 2010.
18. Lin YY, Wei AH, He X, Zhou ZY, Lian S and Zhu W: A comprehensive study of oculocutaneous albinism type 1 reveals three previously unidentified alleles on the TYR gene. Eur J Dermatol 24: 168-173, 2014.

19. Wei AH, Zang DJ, Zhang Z, Yang XM and Li W: Prenatal genotyping of four common oculocutaneous albinism genes in 51 Chinese families. J Genet Genomics 42: 279-286, 2015.

20. Wei A, Wang Y, Long Y, Wang Y, Guo X, Zhou Z, Zhu W, Liu J, Bian X, Lian S and Li W: A comprehensive analysis reveals mutational spectra and common alleles in Chinese patients with oculocutaneous albinism. J Invest Dermatol 130: 716-724, 2010.

21. Anistoroaei R, Fredholm M, Christensen K and Leeb T: Albinism in the American mink (Neovison vison) is associated with a tyrosinase nonsense mutation. Anim Genet 39: 645-648, 2008.

22. Ding B, Ryder OA, Wang X, Bai SC, Zhou SQ and Zhang Y: Molecular basis of albinism in the rhesus monkey. Mutat Res 449: 1-6, 2000.

23. Blaszczyk WM, Arning L, Hoffmann KP and Epplen JT: A Tyrosinase missense mutation causes albinism in the Wistar rat. Pigment Cell Res 18: 144-145, 2005. 